\title{
基于有机化学专业课程培养本科生成就动机的形成
} 以 “对映异构” 教学设计为例

田添禾 ${ }^{2}$, 刘峰 ${ }^{1}$, 冯丽恒 ${ }^{1}$, 张变香 1 ,

1 山西大学化学化工学院, 太原 030006

2 九州大学人间环境学府, 日本福冈

摘要: 以对映异构为例进行了基于专业课程激发学生的学习兴趣, 培养本科生创新与应用能力的教学方法改革; 初 步探索了以专业课程为载体, 通过提高学生在学习中的主体地位形成终身学习的能力和习惯以及引导本科生培养正 确的成就动机的教学设计; 并尝试为高校专业课程的教学增加附加值方面提供有益的参考。

关键词: 成就动机; 专业课程; 对映异构; 教学设计

中图分类号: G64; O6

\section{Aim at the Reform of Organic Chemistry Teaching to Cultivate Students' Achievement Motivation: Taking the "Enantiotopic" Teaching Design as an Example}

\author{
Tianhe Tian ${ }^{2}$, Feng Liu ${ }^{1}$, Liheng Feng ${ }^{1}$, Bianxiang Zhang 1,* \\ ${ }^{1}$ School of Chemistry and Chemical Engineering, Shanxi University, Taiyuan 030006, P. R. China. \\ ${ }^{2}$ Graduate School of Human-Environment Studies, Kyushu University, Fukuoka, Japan.
}

\begin{abstract}
Taking the enantiotopic chapter of organic chemistry as an example, this paper explores the teaching reform to form the ability and habit of lifelong learning by improving students' dominant position in learning and to guide undergraduates to cultivate the right achievement motivation. It also tries to provide useful references for the teaching of specialized courses in colleges and universities.
\end{abstract}

Key Words: Achievement motivation; Specialized course; Enantiotropy; Teaching design

作为一种心理品质, 成就动机 (achievement motivation)是指个体追求自认为重要或有价值的工 作, 并使之达到完美地步的一种内在推动力 ${ }^{[1]}$ 。其特点为: 目标的吸引力越大, 成就动机越大; 个体 施展才干的机会越多, 成就动机越强; 学生的学习成绩与其成就动机呈正相关系。成就动机不仅推 动个体实现自我价值, 也影响着国家社会的发展水平, 甚至与这个国家几年后的经济增长呈显著的 正相关系 ${ }^{[2]}$ 。大学生作为未来社会建设的主力军, 处于心智逐渐走向成熟的人生特殊阶段, 受互联 网、信息技术发展的影响, 其思维方式、行为模式、心理发展、价值观呈现多元化趋势, 进而促使 他们形成多方位的成就动机。因此, 如何正确引导学生感知高等教育过程, 激发他们自我发展的内

收稿: 2020-06-18; 录用: 2020-07-07; 网络发表: 2020-07-16

“通讯作者, Email: zbxthh@sxu.edu.cn

基金资助：山西大学 “课程思政” 示范课程建设项目(202001) 
驱力, 形成良好、正确的成就动机, 使之获得取之不完、用之不竭的前进动力, 感受学习、研究进 步的快乐, 是激发大学生追求上进的一剂良方, 也是大学教师义不容辞的责任, 更是高等教育发展 的一项重要任务。

\section{1 中国、日本大学生 “成就动机” 形成的现状分析}

2019 年, 辛素飞和王一金䣄就中国大学生 1999-2014 年间的成就动机进行了测量分析, 研究结 果表明我国大学生的成就动机水平在这 16 年间显著降低。2020 年日本篠原正典等学者调查分析了 大学生所学课程、自主学习动力及成就动机的相互关系, 明确指出成就动机的培养有助于提升学 习兴趣, 对学生今后的发展益处甚多 ${ }^{[4,5]}$ 。特别是在刚刚升入大学之际, 不少大学生对大学里开放、 自主的多元化教学方式和学习方法不适应, 致使学习的目标缺失, 动力不足, 情绪迷茫, 自制力下 降, 进而产生庈学情绪, 只是追求能够取得学分就满足的小目标, 导致部分学生无法形成比较远大 良好的成就动机 ${ }^{[6]}$ 。因此, 在这拔节孕穗的关键时期, 高校教师应给学生多指点一些迷津, 激发并帮 助学生建立适当的成就动机, 树立进一步追求的目标和梦想, 走出迷茫, 把握当下。特别是在 “两 性一度” (高阶性、创新性与挑战度)的金课理念下，专业课教师应该从打造 “金课” 开始, 推动课堂 改革, 优化课程设置, 丰富教学内容, 培养学生成就动机的形成, 进而产生课程的附加值和升华值, 让大学校园成为梦想起飞的地方, 而不是奋斗的终点。

\section{2 专业有机立体化学课程现状}

有机化合物与人类的日常生活密切相关，有机化学课程是培养化学、化工、制药、生物、环保、 材料等专业人才的重要基础课, 很容易促成学生成就动机的形成 ${ }^{[7,8]}$ 。有机立体化学知识点多、内容 复杂, 特别是概念多且抽象的 “对映异构” 成为众所周知的难教、难学章节, 其中的概念及理论贯 穿于有机化学的后续章节, 特别是在具有生理活性的含杂原子的有机化合物中表现尤为突出 ${ }^{[9,10]}$ 。因 此, 如何在教学过程中巧妙解决学生的理解、记忆问题, 同时在新概念多、理论性强的专业课教学 中, 激发学生的学习兴趣, 培养学生成就动机的形成成为一种新型挑战。古人云: 师者, 传道授业 解惑也; 徒者, 受业质疑悟道也。任何学习过程其实就是对已有知识的理解、保持与迁移来构建新 知识的过程。如果将新知识与学生认知结构中已有的概念、表象及有意义的符号建立内在联系, 学 生就能根据原有的认知来理解、把握新知识的特征与规律。笔者从多年的教学实践中体会到在有机 化学基础理论课程教学中, 若能从不同的角度适当运用学生原有的知识引出新概念, 能够收到事半 功倍的教学效果 ${ }^{[11]}$ 。

\section{3 对映异构章节教学方法改革与实践}

\section{1 教学案例 1: 从光的角度引出有机化合物的旋光性}

光一般被认为是一个物理学范畴的概念, 带给人光明、希望的同时, 为科学研究带来了更多的 灵感。光具有波粒二象性, 即光在不同环境下可表现出不同的特性, 有时为粒子性, 有时则为波动 性。如果将有机化合物放到特定的光的环境中会不会表现出不同的性质? 实验证明某些有机化合物 能使偏振光的振动方向旋转一定角度, 不同物质旋转的角度和方向不同(图 1)。化合物具有的这种性 质称之为该物质的旋光性, 顺时针旋转的为右旋体, 逆时针的为左旋体, 例如组成人体蛋白质的氨 基酸、葡萄糖及乳酸都有旋光性。旋转的角度称之为旋光物质的旋光度 $\alpha$, 并且随溶液的浓度及盛液 管的长度变化而变化。为了区别不同化合物的旋光性, 引入了像物质比重概念一样的比旋光度 $[\alpha]$ 。 比旋光度是旋光性物质的一个物理常数, 能够体现物质的特性, 是质量浓度 $\rho_{\mathrm{B}}=1 \mathrm{~g} \cdot \mathrm{mL}^{-1}$ 的旋光物 质溶液, 放在 $1 \mathrm{dm}$ 长的盛液管中测得的旋光度。通过物理和化学概念的相互引入, 可以使学生深刻 体会到学科之间是相互联系的, 当下所学的知识只要储存在脑子里, 形成一个 “大数据” , 在今后 的学习和工作中自然而然会用到。 


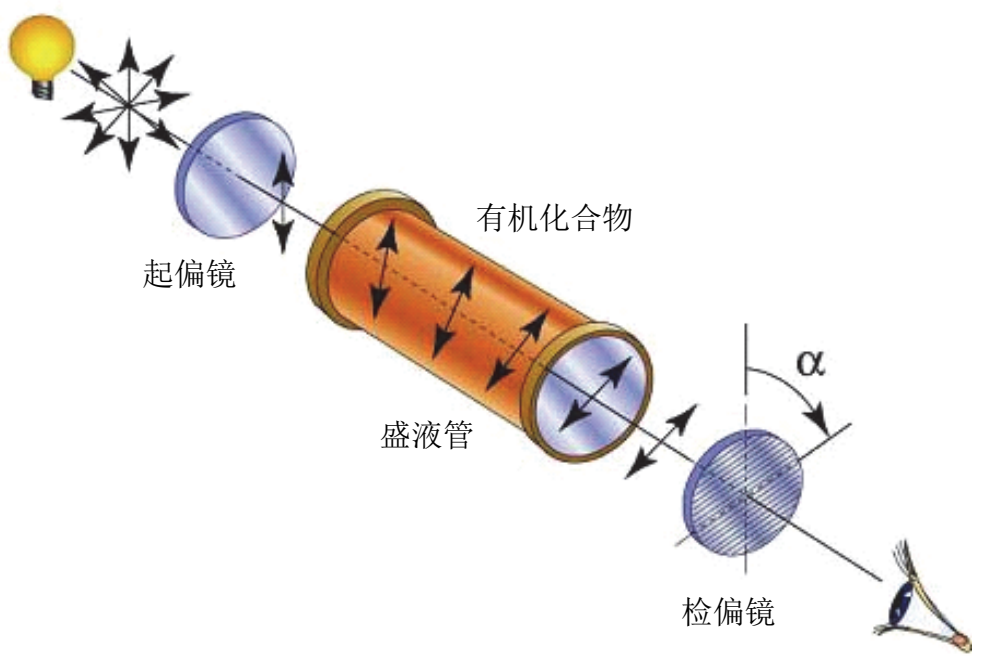

图 1 旋光仪示意图

\section{2 教学案例 2: 从文学作品的角度引出手性碳原子及对映异构体}

《西游记》中, 六耳猕猴和孙悟空站在一起, 上天入地都难以分辨, 最后只有如来佛篎选出真假 悟空, 保证了西行的顺利进行。如来佛就对众神说, 孙悟空和六耳猕猴的差别在 “唯心尔” , 三个 字揭开了两只猴子不同之处。也就是说这两只猴子虽然看起来一样, 但是他们的心不一样, 心里的 想法也不一样, 这让众神恍然明白了修心的重要性。

在有机化合物的分子中，也存在许多像孙悟空和六耳猕猴一样难分彼此的情况，比如图 2 中的 氨基酸, 两个分子的化学式一模一样, 却不能重合, 像是人类的左右手, 存在着镜像关系, 其化学 性质、物理性质均相同, 只有旋光性不同, 而且表现为旋光能力相同, 方向相反, 这就是同分异构 体中的对映异构体, 也叫旋光异构。再比如乳酸也存在一对对映异构体(图 3), 其中连有四个不同基 团的中心碳原子叫做不对称碳原子，也叫手性碳原子，用 $\mathrm{C}^{*}$ 来表示。把四个不同基团按照基团大小 顺序排列, 首先将最小基团放在离观察者的最远处, 然后观察其他三个基团, 如果是按顺时针排列 的, 命名为 $R$-乳酸; 如果是按逆时针排列的, 则命名为 $S$-乳酸, 正所谓乱花渐欲迷人眼, 相不相同 判方知, 核心是 “唯心尔的碳原子”。
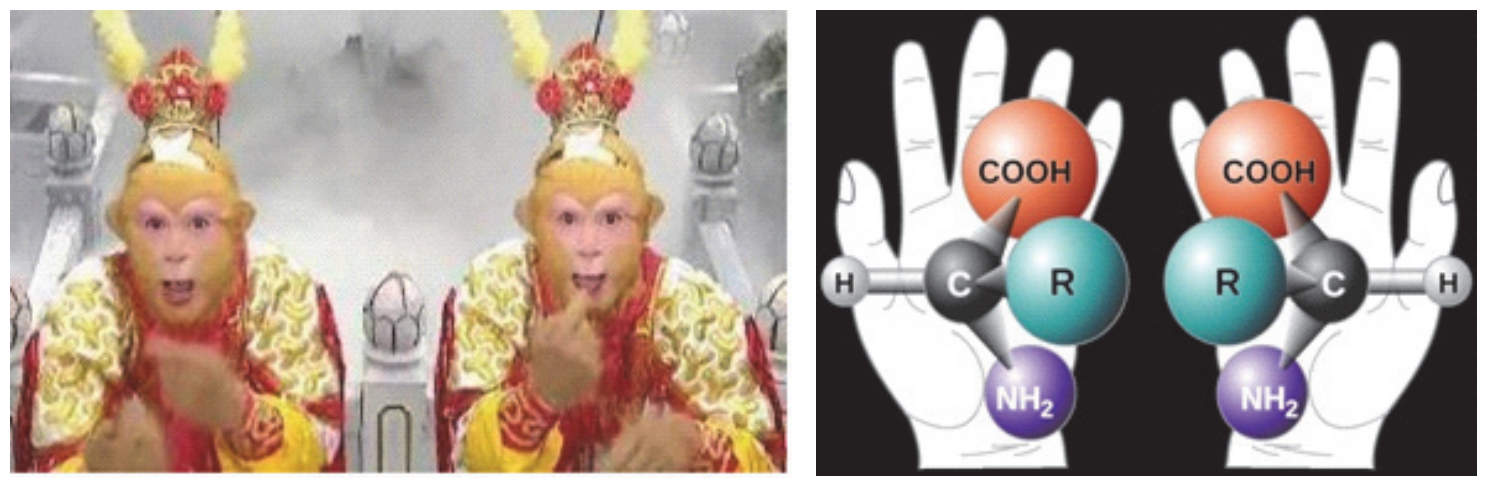

图 2 对映异构体示意图

生命大分子如蛋白质、核酸、多糖等均具有手性, 其基本构成单元亦是如此, 相应的药物和杀 虫剂等分子结构中大多也是含有多个不对称碳原子。例如 2020 年初在治疗新型冠状病毒的药物中 出现的瑞德西韦(Remdesivir), 分子结构中含有 5 个手性碳原子 $C^{*}$ (图 4)。 
<smiles>C[C](O)C(=O)O</smiles>

图 3 乳酸对映异构体

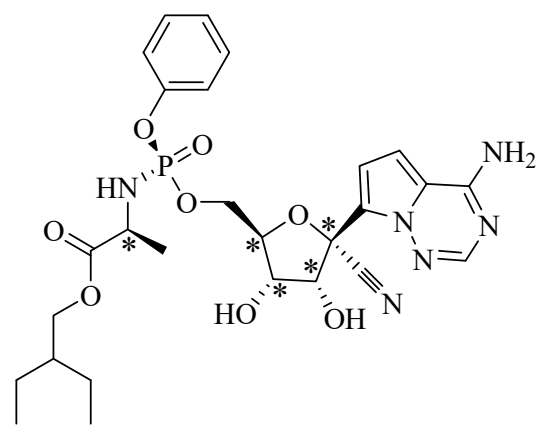

图 4 瑞德西韦结构示意图

\section{3 教学案例 3: 从最美的化学实验中引出外消旋体及前人的科研素养}

2003 年, 由美国科学家发起, 由全球化学家投票评选出了十个 “历史上最美的化学实验”。其中 19 世纪中叶, 巴斯德在显微镜下手工分离右旋和左旋酒石酸盐的实验被评为第一名, 评委给出了较 高的评价(图 5)。巴斯德在优雅简洁的实验室里, 通过单调乏味的手工方式将对映异构体分离, 开拓 了化学结构的新领域。这种对科学的热爱、严谨务实的科研态度及银而不舍的科研作风是值得我们 当代人沉下心来学习的。由此可以说明, 个体追求自认为重要或者有价值的工作时, 内在推动力是 很强大的。

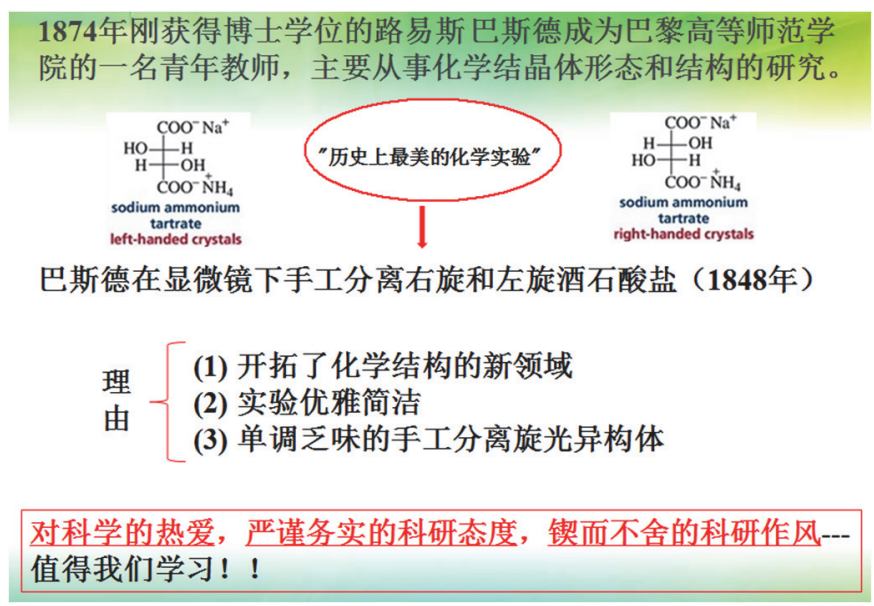

图 5 分离旋光异构体实验教学案例

如果将等摩尔的右旋体和左旋体混合后会形成没有旋光性的外消旋体。因等量的右旋体和左旋 体其旋光能力相同而方向相反, 所以整体表现出旋光度为零。外消旋体是一种混合物, 其性质与相 应的左、右旋体不同, 乳酸的外消旋体写作 $( \pm)$-乳酸或 $(R S)$-乳酸。在此值得注意的是, 构型和旋光方 向之间没有直接联系, $(+) /(-)$ 表示旋光方向, 由实际测量确定, $R / S$ 表示构型, 是以手性碳所连四个 基团在空间的排列来标记，属于系统命名。

\section{4 教学案例 4: 从药物事件中引出对映异构体拆分的重要性}

在生物制药过程中, 经常会制备出手性同分异构体, 而一对对映异构体的生理效用存在较大差 异, 与生理活性的关系主要有以下四种: (1) 生理活性的有与无: 比如左旋氨氯地平能够治疗高血 压, 而右旋氨氯地平却没有效用; (2) 生理活性的强与弱: 普通的莨宕碱指的是由 $R$-茛宕碱和 $S$-莨 宕碱组成的外消旋体, 二者都可以作为抗胆碱药, 但 $R$ 型莨宕碱的作用比后者强 8-50 倍, 作用大毒 性也大。因此, 临床上使用的是 $( \pm)$ 茛宕碱, 取名 “阿托品”。(3) 生理活性的相对抗: 比如依托坐 啉, 左旋体具有利尿作用, 而右旋体具有抗利尿作用。临床上需要拆分得到纯体才能使用, 否则一 
个对映异构体会抵消另一个对映异构体的药效。(4) 生理活性的作用类型、强度均不同: 作为镇静剂 和止吐药物的沙利度胺(反应停), 曾有效治疗早期妊娠中出现的恶心、呕吐, 但服用这种药物的孕妇 产下了双手像海豹前肢一样的畸形婴儿。后来研究发现, 沙利度胺存在两种构型, $S$ 构型的异构体 具有强烈的致畸作用，而 $R$ 构型的异构体不会引起畸变(图 6)。

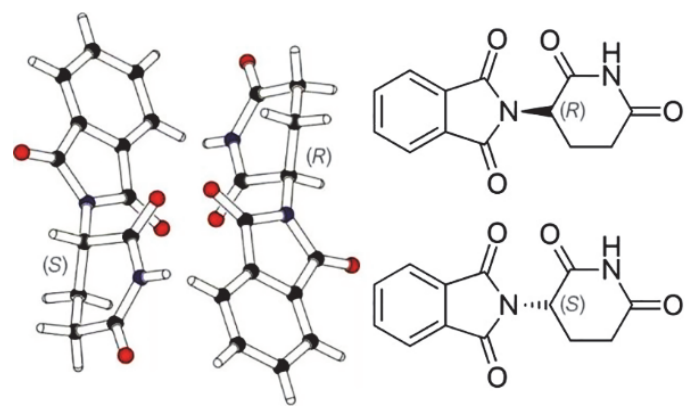

图 6 沙利度胺结构示意图

20 世纪 50 年代德国 “反应停事件”的惨痛教训引起人们对手性药物毒副作用的高度重视。 由此可以说明, 有机化学在人类发展史上功不可没, 但它是一把 “双面刃” 的问题依然存在, 将其 好的一面造福人类, 而将其不利的一面抑制, 避免造成对人类和环境的危害是每个化学工作者应有 的理念。当今这一理念是有机化学课程的配套和延伸, 让学生树立化学不是用来害人的, 而是用来 造福人类的理念, 懂得不讲诚信对自己、家人及社会造成的严重危害, 对培养学生的科学素养、社 会责任感具有极其重要意义。

\section{5 教学案例 5: 从对映异构体拆分的重要性引导学生形成适当的成就动机}

左手性分子和右手性分子就像孙悟空和六耳猕猴一样难以区分和分离, 但是在实际应用中有时 所需要的只是其中一种手性分子, 必须寻找有效的方法来获取单一构型的对映异构体分子。研究学 者们从早期手性的底物控制逐步演化为辅基控制、试剂控制和近年来的催化剂控制。2019 年国际纯 粹与应用化学联合会(IUPAC) 在成立 100 周年纪念日上, 将对映选择性有机催化列为化学领域十大 新兴技术名单。当前聚合物和晶体材料手性分离膜是被寄予厚望的一种解决方案, 但是聚合物膜分 离效率低, 晶体材料又难以制备成膜。因此, 研发一种简便且通用的新型策略来合成并分离一个有 机化合物的所有可能的立体异构体是个相当有难度的挑战, 特别是那些拥有多个相邻立体中心的有 机化合物。通过这些相关的科研报道, 让学生在学习专业课的同时, 了解学科的发展前延、拓展知 识, 引导学生建立所追求的目标, 并积极投身到学习和科研中, 使潜在的创造力得以发挥(图 7)。

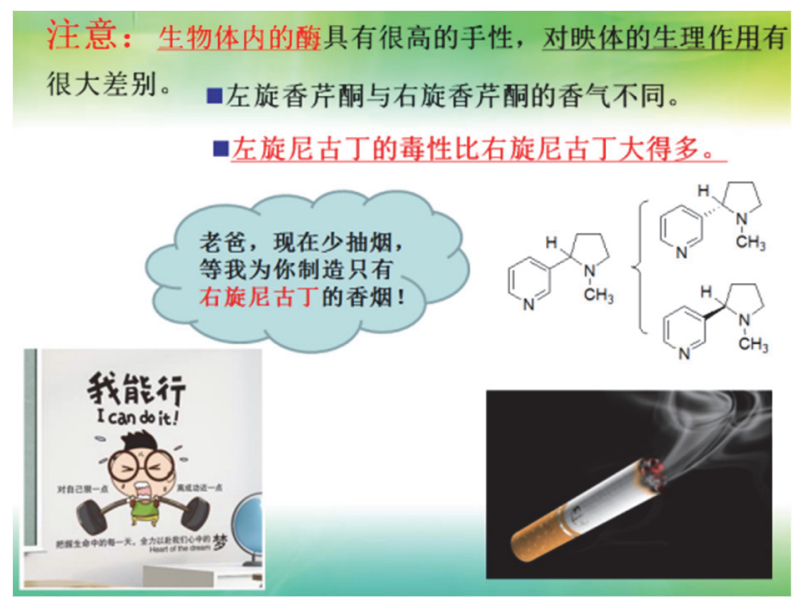

图 7 植物界的对映异构体教学案例 


\section{4 课程实施效果}

通过平时考核、期末考试、师生座谈及问卷调查, 结果表明本课程教学效果有了较大幅度的提 高, 同时提升了对课程的满意度和对任课教师的评价; 大多数学生表示, 今后会持续关注对映异构 体的合成与拆分领域的发展, 愿意将在本课程教学改革中受到的理念和益处迁移到其他专业课程的 学习中去, 使学生初步形成了良好的成就动机; 参与本课程教学改革的这一届学生的作业情况有了 明显提升, 从原来的抄袭答案转变为和老师讨论不同答案的理由。在我校参与有机化学课程学习的 学生人数每学年达到 600 多名, 课程实施中融入成就动机培养的影响范围大、受益面广。

总之, 在传授对映异构专业知识以及其他章节内容的同时, 适当地提炼出理想信念、社会责任 感、科研作风等内在精神, 将培养学生成就动机形成的教学理念融入课程教学中, 会产生课程的附 加值和升华值。随着当今教学风格多样性的开发, 上述内容为笔者在教学实施过程中的一些探索, 希望对其他课程能起到一定的借鉴作用。

\section{参 考 文 献}

[1] [德]莱因贝格. 动机心理学. 王晚蕾, 译. 上海: 上海社会科学院出版社, 2012.

[2] 李科. 成都大学学报(教育科学版), 2007, 21 (11), 21 .

[3] 辛素飞, 王一金理. 心理发展与教育, 2019, No. 3, 288.

[4] 篠原正典. 佛教大学教育学部学会紀要, 2020, 19, 23.

[5] 須藤智, 滑田明暢, 宇賀田栄次. 静岡大学教育研究, 2020, 16, 129.

[6] 张冰融, 宋雪. 现代教育管理, 2014, No. 6, 71.

[7] 那行, 朱亚先. 大学化学, 2019, 34 (8), 25.

[8] 邢其毅, 裴伟伟, 徐瑞秋, 裴坚. 基础有机化学(下册). 第 4 版. 北京: 北京大学出版社, 2017.

[9] 陈神平, 贾春满, 朱文, 王新广. 大学化学, 2019, 34 (4), 20.

[10] 姜新东, 高爽, 于三三, 崔天放, 肖林久. 大学化学, 2020, 35 (6), 28.

[11] 张变香. 大学化学, 2020, 35 (7), 44. 